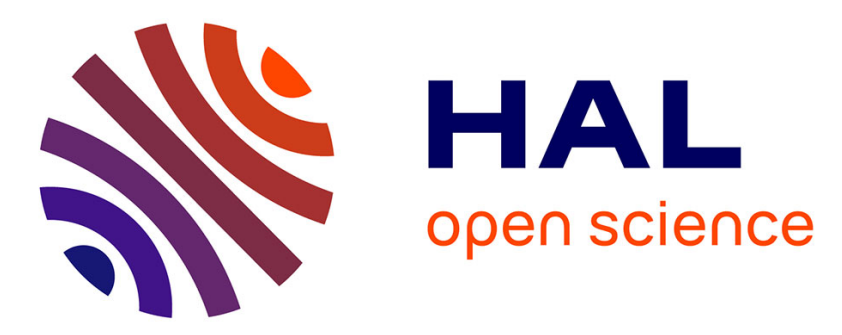

\title{
Interférométrie holographique sur objets diffusants. Application de la mesure du contraste à la détermination des gradients de déplacement
}

\author{
J.C. Charmet, F. Montel
}

\section{- To cite this version:}

J.C. Charmet, F. Montel. Interférométrie holographique sur objets diffusants. Application de la mesure du contraste à la détermination des gradients de déplacement. Revue de Physique Appliquée, 1977, 12 (4), pp.603-610. 10.1051/rphysap:01977001204060300 . jpa-00244215

HAL Id: jpa-00244215

https://hal.science/jpa-00244215

Submitted on 1 Jan 1977

HAL is a multi-disciplinary open access archive for the deposit and dissemination of scientific research documents, whether they are published or not. The documents may come from teaching and research institutions in France or abroad, or from public or private research centers.
L'archive ouverte pluridisciplinaire HAL, est destinée au dépôt et à la diffusion de documents scientifiques de niveau recherche, publiés ou non, émanant des établissements d'enseignement et de recherche français ou étrangers, des laboratoires publics ou privés. 


\title{
INTERFÉROMÉTRIE HOLOGRAPHIQUE SUR OBJETS DIFFUSANTS APPLICATION DE LA MESURE DU CONTRASTE A LA DÉTERMINATION DES GRADIENTS DE DÉPLACEMENT
}

\author{
J. C. CHARMET et F. MONTEL
}

Laboratoire de Mécanique des Milieux Continus, Ecole Supérieure de Physique et Chimie Industrielles 10, rue Vauquelin, 75005 Paris, France

(Reçu le 27 septembre 1976, accepté le 21 décembre 1976)

\begin{abstract}
Résumé. - L'article traite de l'influence de la pupille du système d'observation sur le contraste des franges d'interférométrie holographique dans le cas de la mesure des déformations d'objets diffusants. Après un développement théorique, il est montré comment l'analyse expérimentale des contrastes permet la séparation des paramètres du mouvement et autorise la mesure directe d'un état de déformation plane.
\end{abstract}

\begin{abstract}
The purpose of this paper is to explain the effects of the observation lens aperture size on the contrast of the fringes obtained by holographic interferometry applied to strain measurement on diffusive object surfaces. After theoretical calculations, the authors show how it is possible to separate the motion components or to determine the in-plane strain state of the object surface by use of contrast measurements.
\end{abstract}

1. Introduction. - La géométrie du réseau de franges obtenu, par interférométrie holographique en double exposition, sur un objet diffusant soumis à contraintes et mouvement est décrite par la relation bien connue : $\delta=\left(\mathbf{k}_{1}-\mathbf{k}_{2}\right) \cdot \Delta(Q)=\left\{\begin{array}{cc}2 n \pi & \text { franges brillantes } \\ (2 n+1) \pi & \text { franges sombres }\end{array}\right.$ relation dans laquelle, avec les notations de la figure 1 : $\delta \quad:$ différence de marche entre les deux expositions $\mathbf{k}_{1}$ : vecteur d'onde incident

$\mathbf{k}_{2}$ : vecteur d'onde d'observation

$\Delta(Q)$ : vecteur déplacement du point $Q$ de l'objet $\lambda \quad$ : longueur d'onde de la source d'éclairement

$n \quad$ : nombre entier (positif ou négatif).

Par utilisation de cette relation pour le dépouillement de plusieurs interférogrammes, pris sous des angles de vue différents il est théoriquement possible de mesurer les variations spatiales des trois composantes du vecteur déplacement $\Delta(Q)$ et, par dérivation graphique, numérique ou optique (moirés), de calculer en tout point de la surface de l'objet les composantes du tenseur des déformations. En pratique,

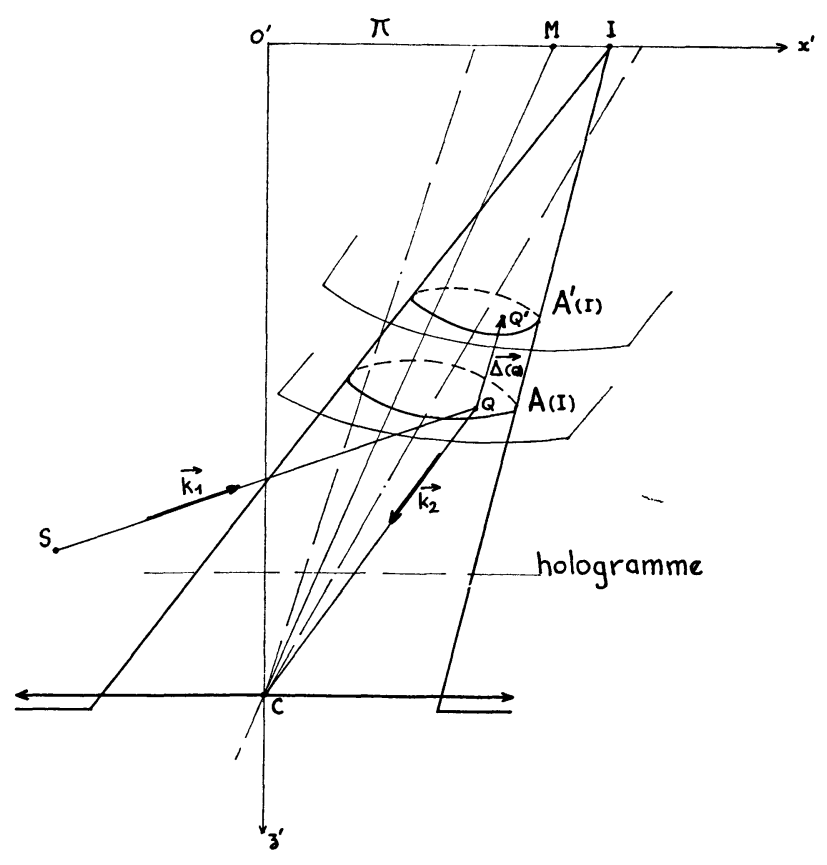

Fig. 1. - Schéma de principe.

[Schematic of holographic optical system.] 
indépendamment de la lourdeur d'un tel dépouillement, l'imprécision sur l'ordre absolu des franges et sur le repérage des franges correspondantes sur les différents interférogrammes conduit à une erreur importante, et parfois même inacceptable, dans la détermination des composantes de déformation.

Mais un interférogramme contient également d'autres informations que celles contenues dans l'équation de géométrie des franges rappelée ci-dessus. Ces informations supplémentaires sont contenues dans l'équation définissant le contraste des franges ou, de façon équivalente, leur localisation spatiale. C'est par le biais de cette équation que nous dégagerons une méthode de mesure plus directe des déformations.

L'obtention de cette équation au contraste nécessite l'introduction de considérations optiques sur le comportement du système d'observation et fait l'objet de la première partie de cet article.

2. Etablissement de la fonction de contraste. L'amplitude lumineuse résultante au point $I$ du plan de visée après double exposition s'écrit à l'aide des équations de la théorie de la diffraction de KirchhoffFresnel :

$$
\begin{aligned}
U(I) \sim \int_{A(I)} & \mathrm{e}^{i \mathbf{k}_{1} \cdot \mathbf{S Q}} a(Q) \mathrm{e}^{i \psi(Q)} \mathrm{e}^{-i \mathbf{k}_{2} \cdot \mathbf{Q I}} \mathrm{d} Q+ \\
& +\int_{A^{\prime}(I)} \mathrm{e}^{i \mathbf{k}_{1} \cdot \mathbf{S} \mathbf{Q}^{\prime}} a\left(Q^{\prime}\right) \mathrm{e}^{i \psi\left(Q^{\prime}\right)} \mathrm{e}^{-i \mathbf{k}_{2} \cdot \mathbf{Q}^{\prime} \mathbf{I}} \mathrm{d} Q^{\prime}
\end{aligned}
$$

relation dans laquelle, avec les notations de la figure 1 :

$A(I) \quad$ est le domaine d'intégration défini par la projection de la pupille du système d'observation sur l'objet, avec $I$ comme centre de projection, lors de la première exposition
$A^{\prime}(I)$ est le domaine d'intégration défini de façon analogue lors de la seconde exposition

$a(Q) \mathrm{e}^{i \psi(Q)}$ est la fonction de diffusion caractérisant la surface de l'objet. $\psi(Q)$ est une phase aléatoire à variation spatiale très rapide.

Après observation à travers le système optique de réponse impulsionnelle $H(I)$, l'intensité recueillie au point $M$ s'écrit (1)

$$
J(M) \sim \iint H(M-I){ }^{*} H(M-J) U(I){ }^{*} U(J) \mathrm{d} I \mathrm{~d} J
$$

relation dans laquelle, sans restreindre la généralité du raisonnement, le grandissement a été pris unitaire afin de simplifier l'écriture en conservant les variables spatiales du plan $\pi$.

Le signe * précédant une expression symbolise l'opération de conjugaison complexe.

L'intégration de cette équation se fait de façon distincte selon que la visée s'effectue sur un plan $\pi$ très proche ou au contraire relativement éloigné de l'objet. Dans le premier cas les aires d'intégration $A(I)$ et $A^{\prime}(I)$ seront négligeables devant l'étendue de la réponse impulsionnelle $H(I)$, dans l'autre, au contraire, l'étendue de $H(I)$ sera négligeable.

2.1 VISÉE SUR UN PLAN PROCHE DE L'OBJET. Dans ce cas les aires d'intégration $A(I)$ et $A^{\prime}(I)$ tendent vers zéro et les points $I$ se confondent avec les points $Q$ de l'objet avant déformation.

En désignant par $\Delta(I)$ le vecteur déplacement du point $Q$ confondu avec $I$ et par $u(I)$ sa projection sur le plan objet, l'intensité en $M$ s'écrit :

$$
\begin{aligned}
J(M) \sim & \int H(M-I) * H(M-J) \mathrm{e}^{i \mathbf{k}_{1} \cdot(\mathbf{I}-\mathbf{J})} a(I) a(J) \mathrm{e}^{i(\psi(I)-\psi(J))} \mathrm{d} I \mathrm{~d} J+ \\
& +\iint H\left(M-I^{\prime}-u\left(I^{\prime}\right)\right)^{*} H\left(M-J^{\prime}-u\left(J^{\prime}\right)\right) \mathrm{e}^{i \mathbf{k}_{1} \cdot\left(I^{\prime}-\mathbf{J}^{\prime}\right)} a\left(I^{\prime}\right) a\left(J^{\prime}\right) \cdot \mathrm{e}^{i\left(\mathbf{k}_{1}-\mathbf{k}_{2}\right)\left(\Delta\left(I^{\prime}\right)-\Delta\left(J^{\prime}\right)\right)} \mathrm{e}^{i\left(\psi\left(I^{\prime}\right)-\psi\left(J^{\prime}\right)\right)} \mathrm{d} I^{\prime} \mathrm{d} J^{\prime} \\
& +\iint H(M-I)^{*} H\left(M-J^{\prime}-u\left(J^{\prime}\right)\right) \mathrm{e}^{i \mathbf{k}_{1} \cdot\left(\mathbf{I}-\mathbf{J}^{\prime}\right)} a(I) a\left(J^{\prime}\right) \cdot \mathrm{e}^{-i\left(\mathbf{k}_{1}-\mathbf{k}_{2}\right) \Delta\left(J^{\prime}\right)} \mathrm{e}^{i\left(\psi(I)-\psi\left(J^{\prime}\right)\right)} \mathrm{d} I \mathrm{~d} J^{\prime} \\
& +\iint H\left(M-I^{\prime}-u\left(I^{\prime}\right)\right)^{*} H(M-J) \mathrm{e}^{i \mathbf{k}_{1} \cdot\left(I^{\prime}-\mathbf{J}\right)} a\left(I^{\prime}\right) a(J) \cdot \mathrm{e}^{i\left(\mathbf{k}_{1}-\mathbf{k}_{2}\right) \Delta\left(I^{\prime}\right)} \mathrm{e}^{i\left(\psi\left(I^{\prime}\right)-\psi(J)\right)} \mathrm{d} I^{\prime} \mathrm{d} J
\end{aligned}
$$

où l'indice' désigne les points homologues (avant déformation) des points de l'objet déformé et le signe * précédant une expression désigne l'opération de conjugaison complexe.

Exprimons le fait que $\psi(I)$ est une phase aléatoire à variation spatiale très rapide en écrivant :

$$
<\mathrm{e}^{i(\psi(I)-\psi(J))}>=\delta(I-J)
$$

$\delta$ étant la fonction de Dirac à deux dimensions.
Cette relation n'est autre que l'expression du principe des rayons homologues : seuls peuvent interférer de façon constructive les rayons issus du même point matériel de l'objet avant et après déformation établi par M. Vienot et généralisé par M. Walles [2].

Faisons l'hypothèse que $a(I)$ et $u(I)$ sont tous deux à variations spatiales lentes devant la dimension de $H(I)$-, ce qui est toujours vérifié avec les ouvertures numériques généralement employées à l'observation. 
$J(M)$ se réduit alors à l'expression :

$$
J(M) \sim 1+\gamma(M) \cdot \cos \left(\left(\mathbf{k}_{1}-\mathbf{k}_{2}\right) \cdot \Delta(M)+\phi(M)\right)
$$

avec $\gamma(M)$ et $\phi(M)$ respectivement module et argument de l'expression complexe :

$$
\frac{\int H(M-u(M)-I)^{*} H(M-I) \mathrm{d} I}{\int|H(M-I)|^{2} \mathrm{~d} I} .
$$

La fonction contraste est liée directement, dans ce cas, à la réponse impulsionnelle du système optique d'observation et au champ de déplacement de la surface de l'objet.

2.2 Visée SUR UN PLAN ÉLOIGNÉ DE L'OBJeT. Dans le cas où le plan d'observation $\pi$ est suffisamment éloigné du plan objet, l'étendue spatiale de la réponse impulsionnelle $H(I)$ est négligeable (tout au moins pour la gamme des ouvertures numériques généralement utilisées à l'observation) devant les aires de $A(I)$ et $A^{\prime}(I)$ de sorte que l'intensité $J(M)$ se simplifie sous la forme :

$$
J(M) \sim U(M)^{*} U(M) .
$$

Nous ferons l'hypothèse que le rayon de courbure de l'objet est suffisamment grand pour que la surface $A$ soit confondue avec le plan tangent à l'objet au point $\mathrm{P}$ projection du centre $\mathrm{C}$ de l'optique d'observation à partir du point $\mathrm{M}$. Les déformations subies par l'objet étant petites, $A^{\prime}$ surface matérielle déduite de $\mathrm{A}$ au cours de la déformation, a une aire égale à celle de $A$. Avec les notations de la figure 2, les points maté-

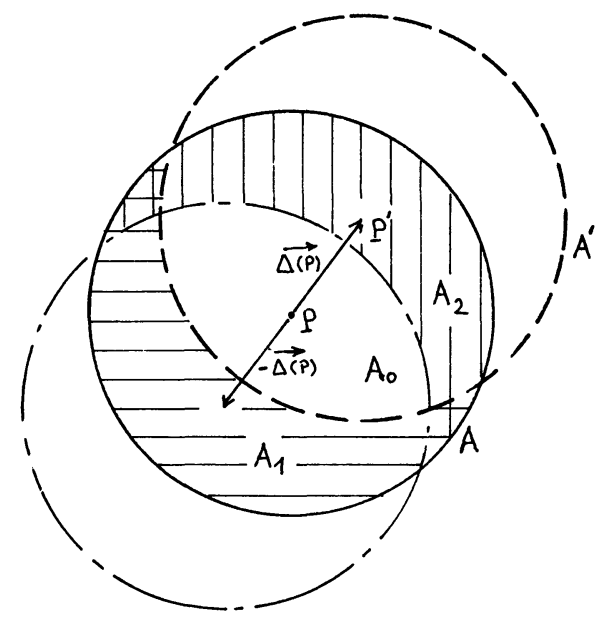

FIG. 2. - Surfaces matérielles et projection de pupille.

[Intersections of the aperture stop projection with the object surfaces before and after sollicitation.]

riels de $A_{2}$ présents dans $A$ à la première exposition, ne le seront plus à la seconde, et inversement les points matériels de $A_{1}$ n'apparaissent dans $A$ qu'à la seconde exposition.
L'amplitude $U(M)$ s'écrira alors :

$$
\begin{aligned}
U(M) \sim \int_{A_{1}+A_{0}} f(Q)+\int_{A_{2}+A_{0}} f\left(Q^{\prime}\right)+ \\
+\int_{A_{1}} f\left(Q^{\prime}\right)+\int_{A_{2}} f(Q)
\end{aligned}
$$

$f(Q)$ représente l'expression

$$
\mathrm{e}^{i\left(\mathbf{k}_{1} \cdot \mathbf{S Q}-\mathbf{k}_{2} \cdot \mathbf{Q M}\right)} a(Q) \mathrm{e}^{i \psi(Q)} \mathrm{d} Q
$$

$f\left(Q^{\prime}\right)$ représente la même expression avec $Q^{\prime}$ au lieu de $Q$ avec $Q$ point courant lors de la première exposition, $Q^{\prime}$ point courant lors de la seconde exposition.

Tenons compte du fait que aire de $A_{1}=$ aire de $A_{2}$ et posons aire de $A_{1}+A_{0}=$ aire de $A_{2}+A_{0}=B$.

Appliquons à nouveau le principe des rayons homologues sous sa forme :

$$
<\mathrm{e}^{i(\psi(I)-\psi(J))}>=\delta(I-J)
$$

$\delta$ étant la fonction de Dirac à deux dimensions.

Enfin supposons que $a(Q)$ est à variation spatiale lente devant la dimension de $A$.

Il en résulte pour l'expression de l'intensité au point M :

$$
J(M) \sim 1+\frac{1}{A} \mathcal{R}\left\{\int_{B} \mathrm{e}^{i \delta \varphi} \mathrm{d} Q\right\}
$$

avec $\mathcal{R}\{$ \} partie réelle de l'expression entre crochets

$$
\delta \varphi=\mathbf{k}_{\mathbf{1}} \cdot\left(\mathbf{S Q}^{\prime}-\mathbf{S Q}\right)-\mathbf{k}_{\mathbf{2}} \cdot\left(\mathbf{Q}^{\prime} \mathbf{M}-\mathbf{Q M}\right)
$$

et $Q^{\prime}$ point homologue de $Q$ au cours de la déformation.

Il reste alors à exprimer $\delta \varphi$ en fonction du champ de déplacement de l'objet et de ses dérivées, entre les deux expositions. Pour ce faire définissons le repère local $\mathrm{P} X Y Z$ tel que, conformément à la figure 3,

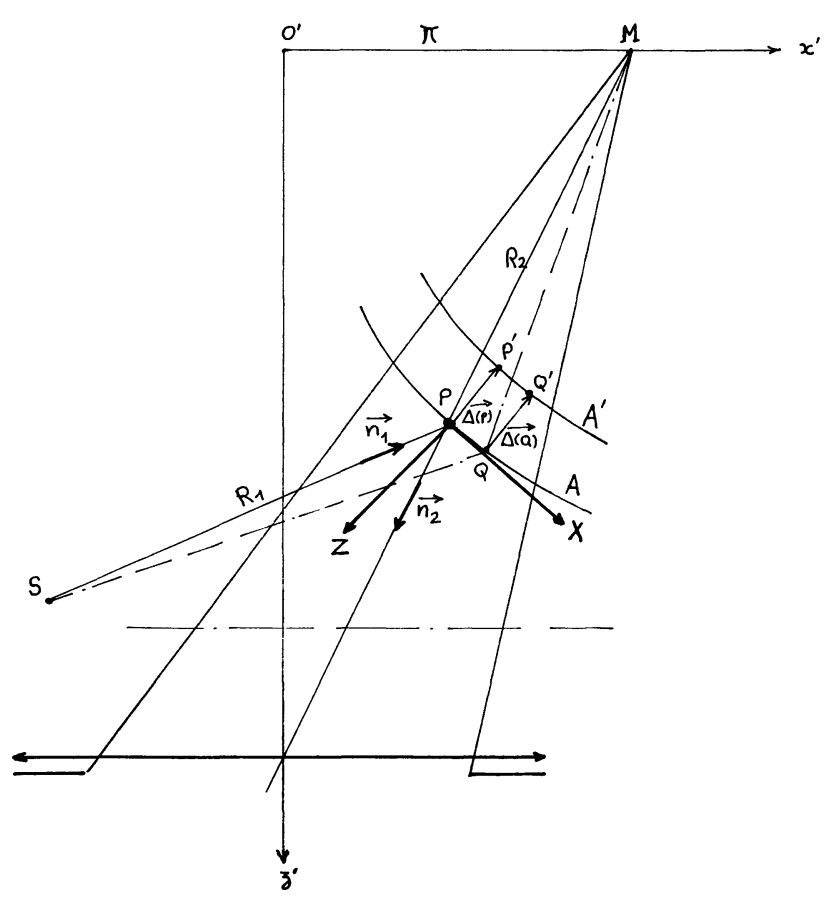

Fig. 3. - Repère local.

[Local coordinate system.] 
l'axe $\mathrm{PZ}$ soit confondu avec la normale en $\mathrm{P}$ à l'objet. Dans ce repère, la relation qui lie les composantes de déplacement de deux points de la zone de projection A s'écrit, au premier ordre :

$$
u_{j}(Q)=u_{j}(\mathrm{P})+u_{j, k} \cdot X_{k}
$$

avec

$$
u_{j, k}=\frac{\partial u_{j}(P)}{\partial X_{k}}
$$

composante du tenseur $\overline{\overline{\text { grad }}} \Delta$ au point $P$ et en appliquant la convention de sommation sur l'indice muet. Par utilisation de cette dernière relation et en effectuant un développement limité au premier ordre des diverses longueurs intervenant dans $\delta \varphi$, afin de les rapporter à celles définies en $\mathrm{P}$.

$J(M)$ s'écrit :

$$
1+\frac{1}{A}\left\{\mathrm{e}^{i \delta_{0}} \cdot \int_{B} \mathrm{e}^{2 \pi i F_{j} X_{j}} \mathrm{~d} B\right\} \quad j=1,2
$$

soit après intégration :

$$
J(M) \sim 1+\frac{B}{A} \cdot\left|B_{n}\left(F_{1}, F_{2}\right)\right| \cdot \cos \left(\delta_{0}+\phi\right)
$$

avec

$$
\delta_{0}=\frac{2 \pi}{\lambda} u_{k} \cdot\left(\alpha_{k}-\beta_{k}\right)
$$

$u_{k}$ composantes du vecteur déplacement du point $\mathrm{P}$ $\alpha_{k}$ composantes du vecteur unitaire porté par SP $\beta_{k}$ composantes du vecteur unitaire porté par PM

et

$$
\begin{aligned}
F_{j}=\frac{1}{\lambda}\left\{u _ { j } \left(\frac{1}{R_{1}}\right.\right. & \left.-\frac{1}{R_{2}}\right)- \\
& \left.-u_{l}\left(\frac{\alpha_{l} \alpha_{j}}{R_{1}}-\frac{\beta_{l} \beta_{j}}{R_{2}}\right)-u_{l, j} \cdot\left(\alpha_{l}-\beta_{l}\right)\right\}
\end{aligned}
$$

$B_{\mathrm{n}}$ transformée de Fourier normalisée de $B$

$\phi$ argument de $B_{\mathrm{n}}$

$j \quad$ indice de sommation prenant seulement les valeurs 1 et 2 .

L'équation $F_{1}=F_{2}=0$ n'est autre que l'équation de localisation spatiale (lieu de contraste maximal) du réseau de franges d'interférences.

Dans de nombreux cas il est possible d'écrire $B \simeq A$ en négligeant la projection sur $A$ du vecteur déplacement de $\mathrm{P}$ devant la dimension de $A$.

La fonction de contraste de l'interférogramme étant établie nous allons l'utiliser, dans cette seconde partie, pour procéder, dans une première expérience, à la séparation des composantes d'un mouvement d'ensemble, puis, dans une seconde expérience, à la détermination de certaines composantes de déformation.
3. Séparation des deux composantes d'un mouvement d'ensemble. - Lorsqu'un objet subit à la fois une translation, d'amplitude $u_{1}$ selon une direction de son plan, et une rotation, d'angle $u_{3,1}$ autour d'un axe de son plan perpendiculaire au précédent, le pas des franges observées est fonction de ces deux quantités. Mais il se trouve que ces deux mouvements donnent lieu à des réseaux de franges semblables: franges parallèles à l'axe de rotation (et perpendiculaires à l'axe de translation) et équidistantes. L'analyse géométrique est donc insuffisante pour séparer les deux composantes du mouvement. L'analyse du contraste fournit l'équation supplémentaire nécessaire à la séparation :

3.1 RECHERCHE DE LOCALISATION. - Les franges ne sont pas localisées sur l'objet et le lieu de meilleur contraste est défini - dans le plan contenant l'axe d'observation et la source d'éclairement, plan d'équation $\alpha_{2}=\beta_{2}=0-$ par l'équation $F_{1}=0$ ce qui donne dans ce cas :

$$
u_{1} \cdot\left(\frac{1-\alpha_{1}^{2}}{R_{1}}-\frac{1-\beta_{1}^{2}}{R_{2}}\right)+u_{3,1} \cdot\left(\alpha_{3}-\beta_{3}\right)=0 .
$$

Une recherche de la localisation fournit donc l'équation supplémentaire. Cependant la détermination précise d'un contraste maximal - qui s'effectue dans une zone où la fonction de contraste a une tangente horizontale - est relativement peu précise et expérimentalement fastidieuse.

3.2 VISÉE SUR LE PLAN OBJET. - Il est beaucoup plus commode d'effectuer une visée sur le plan objet lui-même. Dans ce cas, et pour une pupille d'observation rectangulaire de largeur $2 L$ orientée parallèlement aux franges, un calcul élémentaire, faisant appel aux propriétés des transformées de Fourier, donne pour la fonction de contraste définie par la formule (1)

$$
\gamma(M) \sim \operatorname{sinc} \frac{2 \pi u_{1} L}{\lambda R^{\prime}} \quad \phi(M)=0
$$

où la fonction sinc représente $\sin (x) / x$ et $R^{\prime}$ la distance lentille-objet. Ce résultat signifie simplement que, pour qu'il y ait interférences constructives dans l'image observée, il est nécessaire que la tache d'Airy, associée à l'image d'un point de l'objet avant déplacement, recoupe celle associée au même point matériel après déplacement ; soit, puisque le déplacement correspondant à la rotation $u_{3,1}$ se fait selon l'axe $Z$, que la translation $u_{1}$ soit inférieure à la largeur de la tache d'Airy associée à la pupille d'observation.

L'expérience, conduite avec $u_{1}=10 \mu$ et $u_{3,1}=30^{\prime \prime}$ arc, a donné les résultats repris sur la figure 4 , définissant $u_{1} \cdot u_{3,1}$ est alors calculé à partir de l'équation géométrique donnant le pas des franges. Reprenant cette équation définie dans l'introduction :

$$
\delta=\left(\mathbf{k}_{1}-\mathbf{k}_{2}\right) \cdot \Delta(\mathbf{Q})
$$




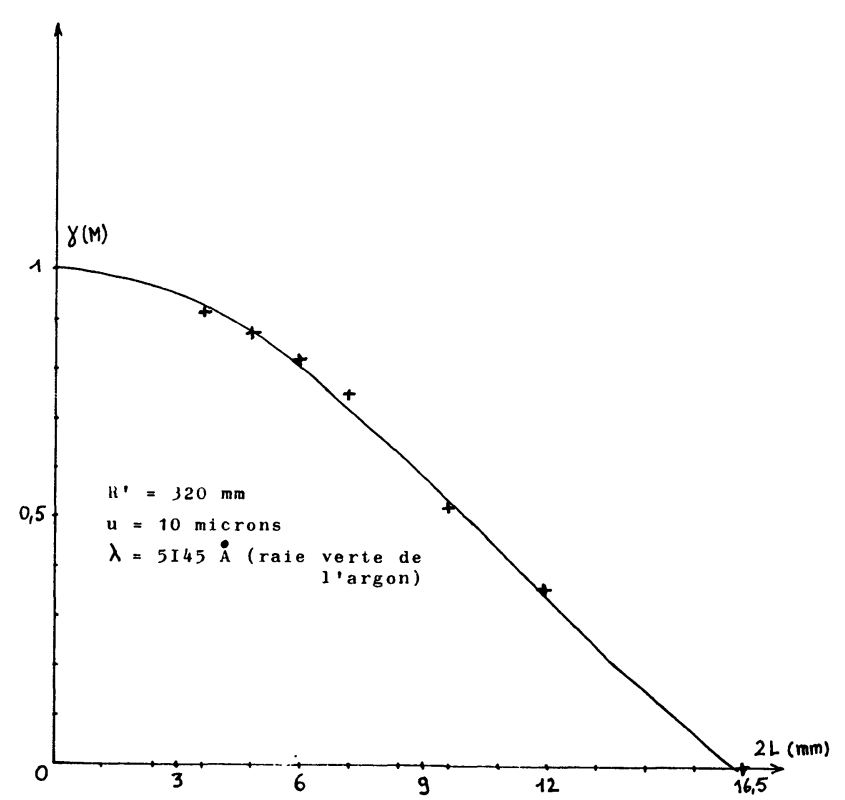

Fig. 4. - Evolution du contraste en fonction du diaphragme.

[Contrast variation as a function of aperture size (Theoretical curve and experimental data).]

l'équation de l'interférogramme devient dans le cas présent :

$$
\delta=\left(\alpha_{1}-\beta_{1}\right) u_{1}+\left(\alpha_{3}-\beta_{3}\right) u_{3,1} X_{1}
$$

où $X_{1}$ désigne l'abscisse du point de visée comptée à partir de l'axe de rotation. Une mesure du pas des franges au voisinage du point de visée - les composantes de $k_{1}$ et $k_{2}$ étant, sauf cas particulier, fonction de ce point - permet alors, connaissant la valeur de $u_{1}$ déterminée par analyse du contraste, de calculer la valeur de la rotation $u_{3,1}$.

Il est d'ailleurs inutile de relever la courbe de contraste. Il suffit de rechercher la largeur $2 L$ de pupille pour laquelle le contraste des franges s'annule. Du fait que la fonction sinc passe à zéro avec une pente non nulle, cette méthode de détermination de $u_{1}$, même dans le cas de relevé effectués à l'œil, est plus précise que celle de la recherche de la localisation (contraste maximal). Une mesure rapide a donné, à l'œil, $u_{1}$ à quelques \% près ; l'erreur étant due au seuil de détection de l'œil et au bruit lumineux accompagnant les franges.

Cette expérience met ainsi en évidence la façon dont il est possible, très rapidement, de séparer des composantes de mouvements complexes, par la simple mesure d'une largeur de fente au point d'annulation du contraste.

Mais, moyennant un dispositif expérimental légèrement plus compliqué, il est possible d'obtenir des informations plus intéressantes encore, allant jusqu'à la détermination directe des déformations par une simple mesure de largeur de fente.

4. Détermination expérimentale des composantes de déformation. - 4.1 ASPECT THÉORIQUE. - Reprenons l'équation de contraste (formule (2)), dans le cas d'une visée loin de l'objet, et développons les expressions de $F_{1}$ et $F_{2}$ :

$$
\begin{array}{r}
F_{1}=\frac{1}{\lambda}\left\{u_{1}\left(\frac{1-\alpha_{1}^{2}}{R_{1}}-\frac{1-\beta_{1}^{2}}{R_{2}}\right)+u_{2}\left(\frac{\alpha_{1} \alpha_{2}}{R_{1}}-\frac{\beta_{1} \beta_{2}}{R_{2}}\right)+\right. \\
+u_{3}\left(\frac{\alpha_{1} \alpha_{3}}{R_{1}}-\frac{\beta_{1} \beta_{3}}{R_{2}}\right)+u_{1,1}\left(\alpha_{1}-\beta_{1}\right) \\
\left.+u_{2,1}\left(\alpha_{2}-\beta_{2}\right)+u_{3,1}\left(\alpha_{3}-\beta_{3}\right)\right\} \\
F_{2}=\frac{1}{\lambda}\left\{u_{2}\left(\frac{1-\alpha_{2}^{2}}{R_{1}}-\frac{1-\beta_{2}^{2}}{R_{2}}\right)+u_{1}\left(\frac{\alpha_{1} \alpha_{2}}{R_{1}}-\frac{\beta_{1} \beta_{2}}{R_{2}}\right)+\right. \\
+u_{3}\left(\frac{\alpha_{3} \alpha_{2}}{R_{1}}-\frac{\beta_{3} \beta_{2}}{R_{2}}\right)+u_{1,2}\left(\alpha_{1}-\beta_{1}\right) \\
\left.+u_{2,2}\left(\alpha_{2}-\beta_{2}\right)+u_{3,2}\left(\alpha_{3}-\beta_{3}\right)\right\}
\end{array}
$$

où $u_{i, j}$ désigne l'expression $\delta u_{i} / \delta X_{j}$ prise au point de percée $P$.

En diaphragmant l'optique d'observation à l'aide d'une pupille de projection rectangulaire, de dimensions $2 L_{x}$ et $2 L_{y}$, respectivement selon l'axe $\mathrm{P} X$ et l'axe P $Y$, la fonction de contraste s'écrit :

$$
B_{\mathrm{n}}(M)=\operatorname{sinc}\left(2 \pi F_{1} \cdot L_{x}\right) \cdot \operatorname{sinc}\left(2 \pi F_{2} \cdot L_{y}\right) .
$$

En choisissant de plus un montage tel que

$$
R_{1}=R_{2}=\infty
$$

(éclairage parallèle et observation au foyer d'une lentille) nous avons réalisé l'analogue d'une rosette, associée à un détecteur de pente. En effet, tous les termes contenant les composantes du vecteur déplacement du point visé s'annulent, seules interviennent les composantes du tenseur gradient de déplacement défini dans le repère local associé au point de visée. L'interférométrie holographique est alors affranchie du repère fixe $O x y z$ qui rend difficile l'analyse géométrique directe des déplacements. Le contraste ne dépend plus que des composantes de déformation dans le repère local $\mathrm{P} X Y Z$ associé au point de visée.

Pour un même point de visée $P$ sur l'objet, réalisons la succession d'opérations suivante :

a) Choisissons $\alpha_{2}=\beta_{2}=0 ; \quad F_{\mathrm{n}}$ s'écrit alors :

$$
\begin{aligned}
B_{\mathrm{n}}= & \operatorname{sinc}\left\{\frac{2 \pi}{\lambda}\left(u_{1,1}\left(\alpha_{1}-\beta_{1}\right)+u_{3,1}\left(\alpha_{3}-\beta_{3}\right)\right) L_{X}\right\} \times \\
& \times \operatorname{sinc}\left\{\frac{2 \pi}{\lambda}\left(u_{1,2}\left(\alpha_{1}-\beta_{1}\right)+u_{3,2}\left(\alpha_{3}-\beta_{3}\right)\right) L_{y}\right\} .
\end{aligned}
$$

En rendant $L_{x}$ suffisamment petit, pour que la première fonction sinc soit quasiment égale à 1 , la détermination de la largeur $L_{y}$ annulant le contraste donnera la valeur de :

$$
u_{1,2}\left(\alpha_{1}-\beta_{1}\right)+u_{3,2}\left(\alpha_{3}-\beta_{3}\right) \text {. }
$$


Réciproquement, rendons $L_{y}$ petit et annulons le contraste avec $L_{x}$, on obtient la valeur de :

$$
u_{1,1}\left(\alpha_{1}-\beta_{1}\right)+u_{3,1}\left(\alpha_{3}-\beta_{3}\right)
$$

$u_{3,1}$ et $u_{3,2}$ sont facilement déterminés, soit par dérivation du réseau de franges, soit par annulation du contraste, avec la configuration particulière $\alpha_{1}=+\beta_{1}$ (observation dans la direction de réflexion spéculaire.

b) Répétons des opérations analogues avec

$$
\alpha_{1}=\beta_{1}=0
$$
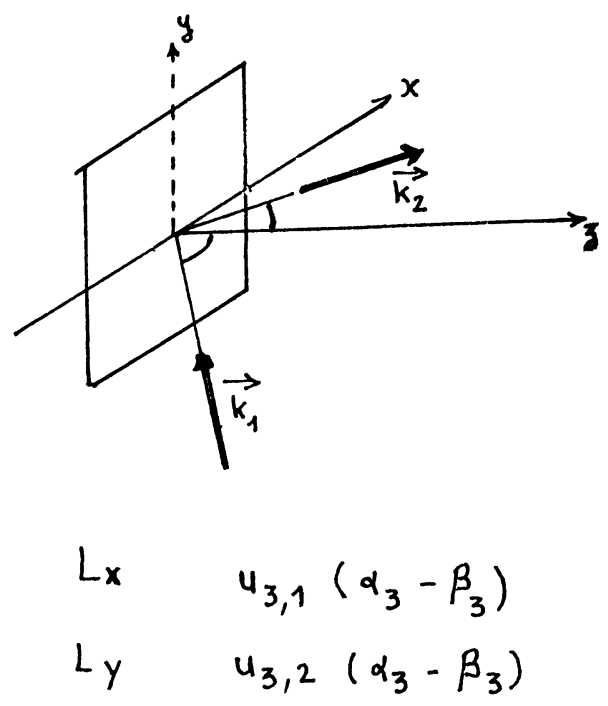

3

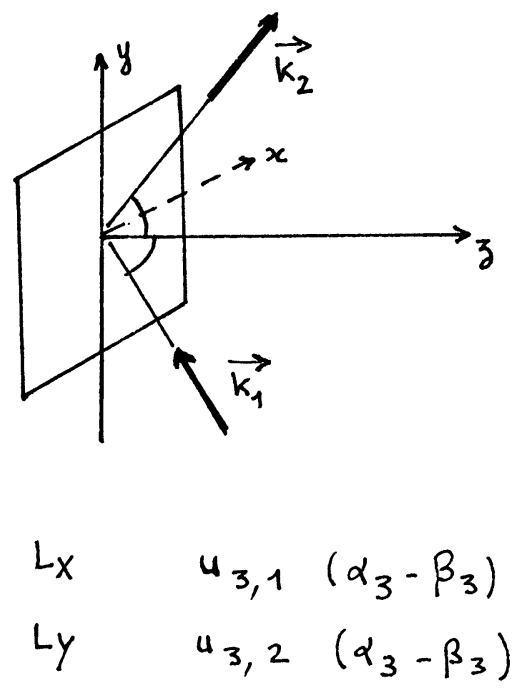

en jouant sur $\alpha_{2}$ et $\beta_{2}$, on obtient de façon identique les valeurs de $u_{2,1}$ et $u_{2}$.

Ainsi, par l'intermédiaire de quatre interférogrammes, et grâce à l'analyse du contraste, il est possible de déterminer, point par point, et sans être gêné par les composantes de déplacement:

- les pentes locales de la déformée : $u_{3,1}$ et $u_{3,2}$,

- l'état de déformation plane :

$$
\varepsilon_{11}=u_{1,1} \varepsilon_{22}=u_{2,2} \varepsilon_{12}=\varepsilon_{21}=\frac{1}{2}\left(u_{1,2}+u_{2,1}\right) \text {. }
$$

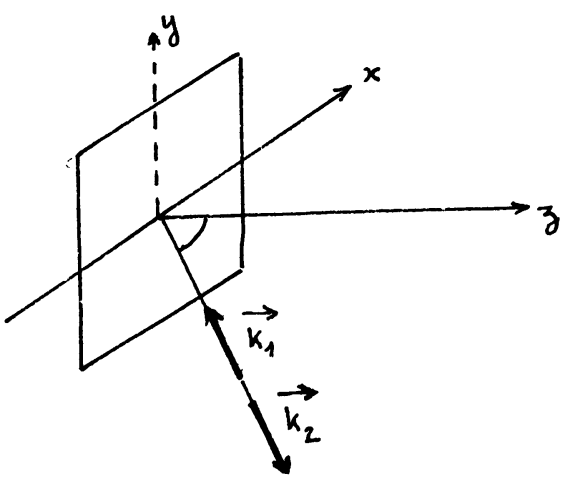

$L_{x} u_{1,1}\left(\alpha_{1}-\beta_{1}\right)+u_{3,1}\left(\alpha_{3}-\beta_{3}\right)$

Ly $u_{1,2}\left(\alpha_{1}-\beta_{1}\right)+u_{3,2}\left(\alpha_{3}-\beta_{3}\right)$

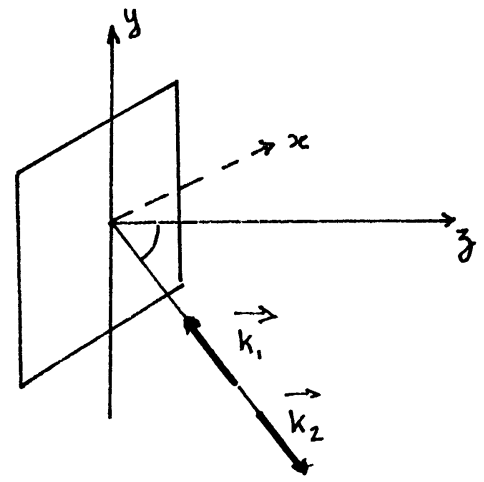
$L_{x} \quad u_{2,1}\left(\alpha_{2}-\beta_{2}\right)+u_{3,1}\left(\alpha_{3}-\beta_{3}\right)$
Ly $u_{2,2}\left(\alpha_{2}-\beta_{2}\right)+u_{3,2}\left(\alpha_{3}-\beta_{3}\right)$

LES CUNFIGURATIONS 1 et 3 SUNT EQUIVALENTES LORSQUE $\overrightarrow{\mathrm{k}}_{1}$ EST L'IMAGE de $\vec{K}_{2}$ PAR RAPPORT AU PLAN OBJET

Fig. 5. - Schéma des quatre configurations de mesure. [Schematic of the four experimental arrangments.] 
Cette procédure se résume par les quatre schémas de la figure 5, représentant les conditions expérimentales de réalisation des quatre interférogrammes. Sur chaque configuration sont portées les combinaisons des composantes du gradient de déplacement accessibles à la mesure.

Cependant, comme pour l'analyse des déplacements, les termes $u_{1,1}$ et $u_{2,1} ; u_{2,2}$ et $u_{1,2}$ apparaissent toujours en liaison avec $u_{3,1}$ et $u_{3,2}$. Pour augmenter la sensibilité, il faudra donc choisir un montage tel que $\beta_{1} \simeq-\alpha_{1}$ et $\left|\alpha_{1}\right|$ grand, ce qui nécessite l'emploi d'un revêtement rétroréflecteur sur l'objet, la direction d'observation étant voisine de celle d'éclairement.

La dynamique de mesure est limitée :

- vers les faibles déformations par la dimension de la lentille d'observation,

- vers les grandes déformations par la taille du grain de speckle (faibles ouvertures) comparée au pas des franges.

4.2 RÉSUltats EXPÉRIMENTAUX PRÉLIMINAIRES. L'expérience décrite ici et réalisée rapidement, à titre de vérification préliminaire, a donné des résultats encourageants et des ordres de grandeur cohérents.

La figure 6 représente le montage optique et l'éprouvette de flexion sur appui simple, chargée en son
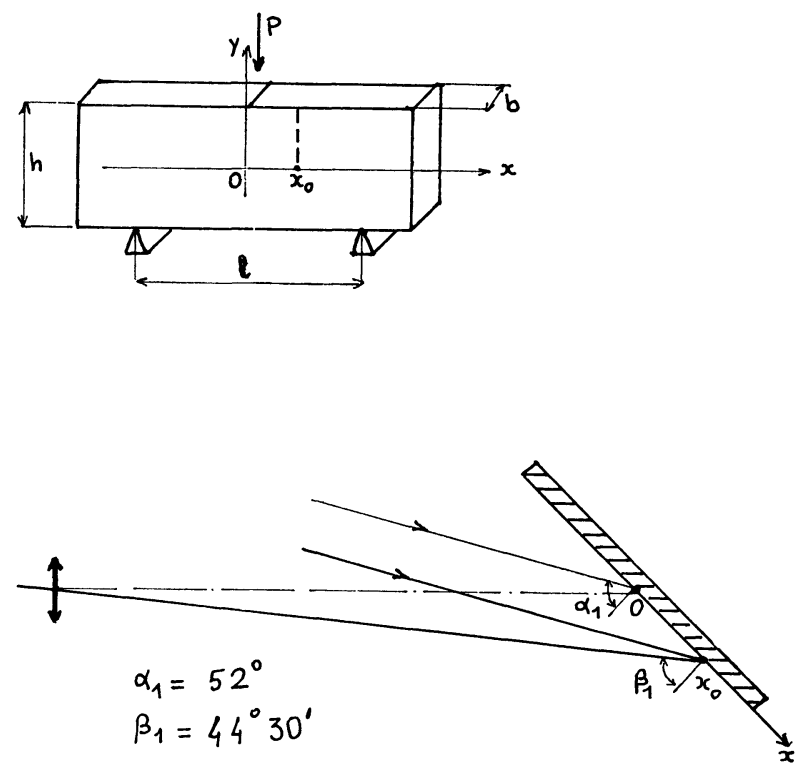

Fig. 6. - Schéma de l'éprouvette et du montage optique.

[Plexiglass bar under bending and optical arrangement.]

centre. Le montage mécanique de flexion était conçu de sorte que les composantes $u_{3,1}$ et $u_{3,2}$ restent négligeables et le montage optique donnait directement accès à $u_{1,1}=\varepsilon_{11}$ dans ces conditions.

Loin des zones de Saint-Venant, la résistance des matériaux donne pour les variations spatiales de $\varepsilon_{11}$ :

$$
\varepsilon_{11}=\frac{P}{2 E I_{z}}\left(\frac{l}{2}-|x|\right) y \text { avec } I_{z}=\frac{b h^{3}}{12} .
$$

Les points de la figure 7 représentent l'évolution de $\varepsilon_{11}$ relevée expérimentalement, le long d'un axe d'équation $x=x_{0}$ situé hors de la zone de SaintVenant, pour deux charges $P$ et $2 P$.

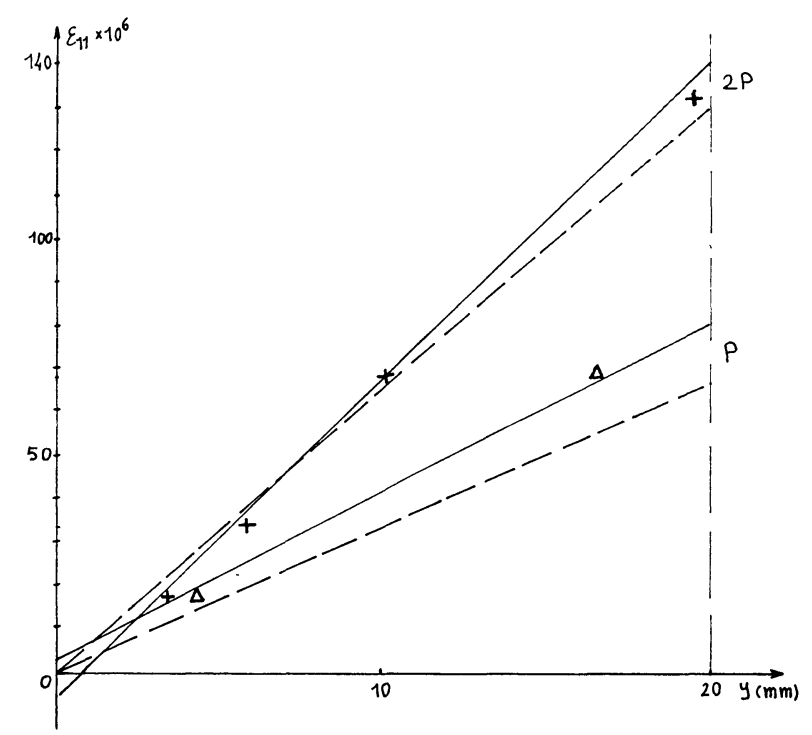

Fig. 7. - Résultats expérimentaux. En pointillé : évolution calculée; En traits pleins : courbe expérimentale obtenue par régression.

[Experimental results : dotted lines : calculated curves; solid lines : experimental data.]

Les courbes en trait plein représentent les droites de régression linéaire associées aux points expérimentaux.

Les courbes en pointillés correspondent aux variations de $\varepsilon_{11}$ calculées à partir de la formule ci-dessus avec les valeurs suivantes :

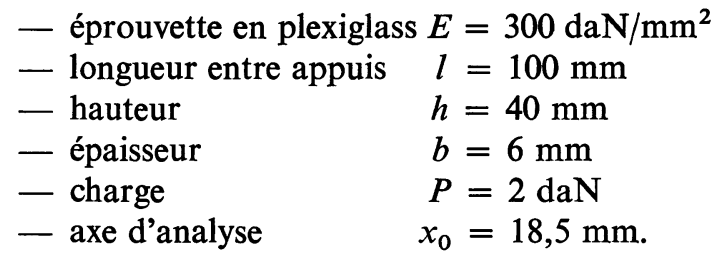

Dans cet essai préliminaire rapide, les dépouillements liés à l'analyse du contraste sont encore longs et fastidieux. Il est en effet nécessaire de procéder à une estimation aussi précise que possible de la fonction de bruit pour calculer la valeur absolue du contraste (valeur de la fonction de contraste en l'absence de bruit) qui seule permet l'application des formules établies plus haut. Aussi, avons-nous été contraints de limiter le nombre des points expérimentaux, d'autant que nous sommes actuellement occupés à la réalisation d'un montage utilisant une méthode de zéro dont la description fait l'objet du paragraphe suivant et grâce à laquelle nous pensons améliorer sérieusement les performances de la méthode décrite, tout en réduisant considérablement le temps de dépouillement. 
4.3 LA MÉTHODE DE ZÉRO DE CONTRASTE. - La mesure du contraste en un point nécessite la détermination du rapport :

$$
\frac{I_{\max }-I_{\min }}{I_{\max }+I_{\min }} \text { en ce point . }
$$

En réalité les valeurs de $I_{\max }$ et $I_{\min }$ sont mesurées sur les franges brillantes et noires voisines du point de mesure. Cela introduit une erreur due au fait que ces valeurs ne correspondent pas exactement à celles du point de visée. D'autre part il est nécessaire d'estimer le bruit afin de calculer le contraste en valeur absolue.

Afin d'améliorer la précision et de simplifier les dépouillements, nous réalisons actuellement le montage schématisé par la figure 8 , reprenant la technique
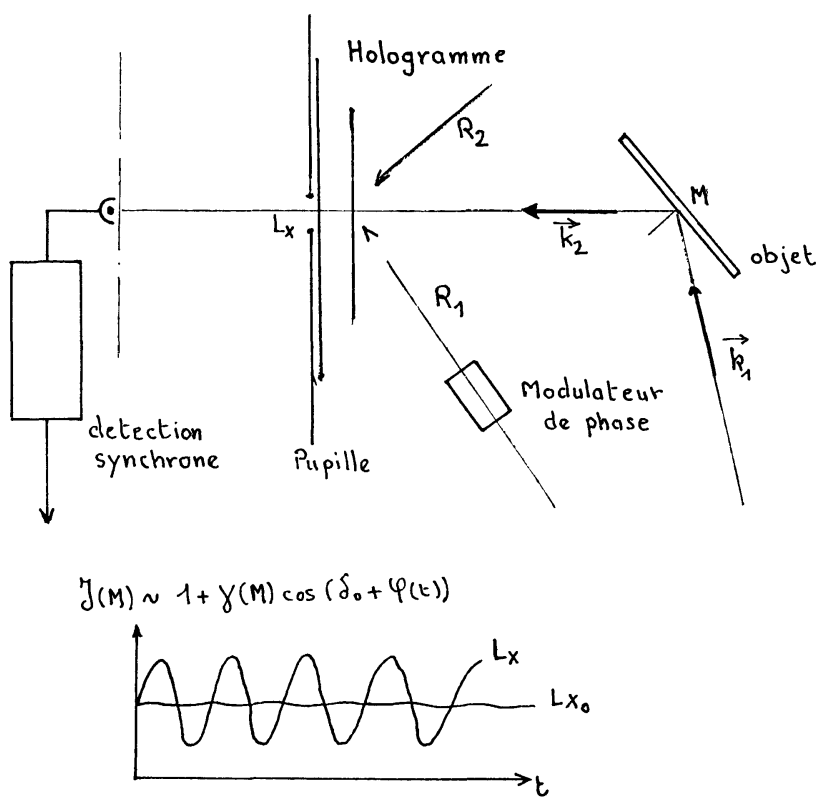

Fig. 8. - Méthode de zéro de contraste. [Two-reference-beam holography.]

des hologrammes en double exposition décrite par Dandliker, Marom et Mottier [3]. L'enregistrement holographique de chaque état mécanique de l'objet est lié à une référence $R_{1}$ ou $R_{2}$. La lecture simultanée de l'hologramme par $R_{1}$ et $R_{2}$ permet d'ajuster la phase relative de l'image d'un état par rapport à l'autre, donc aussi la phase origine de l'interférogramme, en agissant sur la phase d'une des références.
L'équation générale de l'interférogramme s'écrit alors :

$$
J(M) \sim 1+\gamma(M) \cos \left(\delta_{0}+\varphi(t)\right)
$$

avec :

$\gamma(M)$ fonction de contraste

$\delta_{0} \quad$ équation géométrique des franges

$\varphi(t)$ modulation de phase d'une des références.

L'association d'une modulation linéaire de phase et d'une détection synchrone permet alors la mesure du contraste au point de visée tout en réduisant considérablement l'influence du bruit. Il suffit alors de faire varier la largeur de la pupille fente d'analyse jusqu'à annuler la composante alternative détectée, c'est-à-dire le contraste, pour ramener la méthode à une méthode de zéro conduisant, pour le dépouillement, à la simple résolution d'un système d'équations linéaires :

$$
B_{\mathrm{n}}=0 \text { soit }\left\{\begin{array}{l}
F_{1} \cdot L_{x}=\pi \\
F_{2} \cdot L_{y}=\pi
\end{array}\right.
$$

pour la première annulation de contraste.

Nous attendons de sérieuses améliorations par cette méthode grâce à la réduction du bruit et à la détection de zéro. Ses performances feront l'objet d'un article ultérieur.

5. Conclusion. - L'analyse des interférogrammes, sous l'aspect contraste, offre grâce à l'établissement de la fonction de contraste, des possibilités supérieures à celles de la simple analyse géométrique. Moyennant un montage expérimental plus complexe et certaines précautions (tel un revêtement rétroréflecteur), l'interférométrie holographique devient un véritable outil extensométrique indépendant des déplacements du point d'analyse, au même titre que les jauges de contraintes, et doué d'une sensibilité voisine (quelques $10^{-6}$ ). Les résultats obtenus au cours de cette expérience préliminaire sont prometteurs et nous espérons améliorer la précision par la mise en œuvre de la méthode de zéro de contraste.

Rermerciements. - Nous tenons à remercier vivement Monsieur Jean Ebbeni, Professeur à l'Université Libre de Bruxelles, pour les discussions et les encouragements qu'il nous a prodigué au cours de ces travaux.

\section{Bibliographie}

[1] Goodman, J. W., Introduction à l'Optique de Fourier et à l'Holographie (Masson et Cie) 1972, Chapitre 6: Analyse fréquentielle des systèmes optiques formant des images. Cas de l'éclairage cohérent, pp. 101-103.

[2] Walles, S., On the concept of homologous rays in interfe- rometry of diffusely reflecting surfaces. Opt. Acta 17 (1970) pp. 899-913.

[3] Dandliker, R., Marom, E. and Mottier, F. M., Tworeference-beam holographic interferometry. J. Opt. Soc. Am. 66 (1976) 23-30. 